ARTICLE

https://doi.org/10.1038/s41467-019-12676-7

\title{
High-speed domain wall racetracks in a magnetic insulator
}

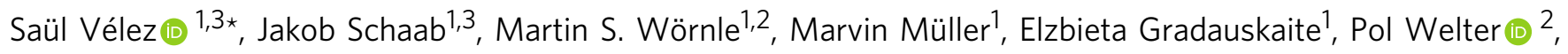
Cameron Gutgsell ${ }^{1}$, Corneliu Nistor ${ }^{1}$, Christian L. Degen (1) ${ }^{2 \star}$, Morgan Trassin (1) ${ }^{1 \star}$, Manfred Fiebig (1) ${ }^{1 \star} \&$ Pietro Gambardella (1* ${ }^{1 \star}$

Recent reports of current-induced switching of ferrimagnetic oxides coupled to heavy metals have opened prospects for implementing magnetic insulators into electrically addressable devices. However, the configuration and dynamics of magnetic domain walls driven by electrical currents in insulating oxides remain unexplored. Here we investigate the internal structure of the domain walls in $\mathrm{Tm}_{3} \mathrm{Fe}_{5} \mathrm{O}_{12}$ ( $\mathrm{TmlG}$ ) and $\mathrm{TmlG} / \mathrm{Pt}$ bilayers, and demonstrate their efficient manipulation by spin-orbit torques with velocities of up to $400 \mathrm{~ms}^{-1}$ and minimal current threshold for domain wall flow of $5 \times 10^{6} \mathrm{~A} \mathrm{~cm}^{-2}$. Domain wall racetracks are defined by Pt current lines on continuous TmIG films, which allows for patterning the magnetic landscape of TmIG in a fast and reversible way. Scanning nitrogen-vacancy magnetometry reveals that the domain walls of $\mathrm{TmIG}$ thin films grown on $\mathrm{Gd}_{3} \mathrm{Sc}_{2} \mathrm{Ga}_{3} \mathrm{O}_{12}$ exhibit left-handed Néel chirality, changing to an intermediate Néel-Bloch configuration upon Pt deposition. These results indicate the presence of interfacial Dzyaloshinskii-Moriya interaction in magnetic garnets, opening the possibility to stabilize chiral spin textures in centrosymmetric magnetic insulators.

\footnotetext{
${ }^{1}$ Department of Materials, ETH Zurich, 8093 Zurich, Switzerland. ${ }^{2}$ Department of Physics, ETH Zurich, 8093 Zurich, Switzerland. ${ }^{3}$ These authors contributed equally: Saül Vélez and Jakob Schaab. *email: saul.velez@mat.ethz.ch; degenc@ethz.ch; morgan.trassin@mat.ethz.ch; manfred.fiebig@mat.ethz. ch; pietro.gambardella@mat.ethz.ch
} 
S: pintronics relies on the use of current-induced torques for manipulating the magnetization of thin films and nanodevices ${ }^{1}$. Owing to spin-orbit coupling, charge currents flowing in heavy metals, such as $\mathrm{Pt}$, $\mathrm{Ta}$, or $\mathrm{W}$, generate spin currents that exert a torque onto an adjacent ferromagnetic layer ${ }^{2,3}$. These so-called spin-orbit torques (SOTs) are capable of reversing the magnetization of ferromagnets in a highly efficient and ultra-fast manner ${ }^{4-8}$, as well as driving domain walls (DWs) at very high velocities ${ }^{9-11}$. Most studies in this area, however, have been performed on ultrathin metallic ferromagnets, for which extensive characterizations of the DW structure and velocity have been reported ${ }^{12-20}$.

Magnetic insulators offer exciting perspectives for spintronic and magnonic applications beyond conventional metallic systems ${ }^{21}$. In particular, ferrimagnetic rare-earth garnets coupled to heavy metal layers have attracted attention due to the possibility of electrically exciting and detecting propagating magnons ${ }^{22-25}$, as well as for their low-power and high-frequency magnetization dynamics $^{26}$. Despite increasing interest in such systems, however, the electrical manipulation of the equilibrium magnetization has not been investigated in detail. Current-induced switching of magnetic insulators has been only recently demonstrated in $\mathrm{Tm}_{3} \mathrm{Fe}_{5} \mathrm{O}_{12}$ (TmIG) and $\mathrm{BaFe}_{12} \mathrm{O}_{19}$ in combination with either Pt or $\mathrm{W}$ layers ${ }^{27-30}$. These studies relied on magnetoresistance measurements to detect the orientation of the magnetization, from which the dynamics of the switching process cannot be inferred.

In this work, we present a combined scanning nitrogen-vacancy (NV) magnetometry and spatially resolved magneto-optic Kerr effect (MOKE) study of the DW structure and dynamics driven by SOTs in racetrack structures embedded in a TmIG layer. We demonstrate highly efficient current-induced DW motion in TmIG/Pt, with mobility comparable or larger than metallic ferromagnets, a remarkable low threshold for DW flow, and very small depinning fields. We further provide a direct characterization of the DW width and internal structure in thin-film TmIG and TmIG/Pt bilayers. Previous studies in garnets were only able to provide estimates of the DW width based on indirect or diffraction-limited optical measurements, with reported values ranging from tens of nanometers to micrometers ${ }^{31-34}$. Scanning NV magnetometry reveals that the DWs in TmIG films are only $\sim 20 \mathrm{~nm}$ wide and have a well-defined chiral structure, which changes from the left Néel in TmIG to intermediate left Néel-Bloch in TmIG/Pt. Given that the crystal structure of TmIG is centrosymmetric, these findings evidence the presence of strong interfacial Dzyaloshinskii-Moriya interaction (DMI) in TmIG grown on substituted gadolinium gallium garnet $\mathrm{Gd}_{3} \mathrm{Sc}_{2} \mathrm{Ga}_{3} \mathrm{O}_{12}$ (SGGG), which is attenuated by the deposition of Pt. The DMI is the key ingredient required to stabilize chiral Néel DWs in ferromagnets and ferrimagnets with perpendicular magnetization, which can then be driven by SOTs at very high velocities ${ }^{10-15}$. In contrast with metallic ferromagnets, TmIG thin films support the formation of Néel DWs without introducing heavy metal layers. Our results show that ferrimagnetic garnets are ideal materials for fabricating efficient and high-speed DW racetracks.

\section{Results}

Local switching in continuous TmIG films. TmIG $(8 \mathrm{~nm}) / \mathrm{Pt}$ $(5 \mathrm{~nm})$ bilayers were grown on SGGG (111)-oriented substrates by a combination of pulsed laser deposition for epitaxial growth of the garnet and in-situ dc sputtering for $\mathrm{Pt}$. The numbers between parentheses indicate the thickness of each layer. $\mathrm{Pt}$ current lines were patterned in the shape of Hall bars by optical lithography and etching of the metal, leaving a continuous TmIG film (see Methods). Figure la shows an optical image of a TmIG/ Pt device. The structural, topographic, magnetic, and electric characterization of the TmIG film and the TmIG/Pt bilayer are reported in the Supplementary Notes 1-3.

The magnetic state of the TmIG film underneath the Pt current line, $+\mathbf{m}$ (up) or $-\mathbf{m}$ (down), can be read electrically by measuring the transverse Hall resistance $R_{x y}$, as shown in Fig. $1 \mathrm{~b}$ during a sweep of the out-of-plane magnetic field $H_{z}$. The measurement confirms that the films exhibit robust perpendicular magnetic anisotropy with a coercive field of about 40 Oe. In agreement with a previous report ${ }^{27}$, the magnetization of TmIG can be deterministically switched upon the application of a current pulse of sufficient current density $J_{x}$ in the presence of a constant in-plane field $H_{x}$ (Fig. 1c). The switching polarity is determined by the damping-like component of the $\mathrm{SOT}^{2-4}$, which stabilizes $+\mathbf{m}$ for $J_{x}$ parallel to $H_{x}$ and $-\mathbf{m}$ for $J_{x}$ antiparallel to $H_{x}$ in TmIG/Pt. Notably, we found that full switching can be achieved with pulses of $1 \mathrm{~ms}$ at current densities below $10^{7} \mathrm{~A} \mathrm{~cm}^{-2}$ for an in-plane field as small as $\left|H_{x}\right|=20 \mathrm{Oe}$, which confirms the high quality of our devices (see also Supplementary Note 3 ).

A distinctive feature of our experiments is that TmIG covers the entire substrate, but switching occurs only in the region defined by the Pt current line. This is clearly seen in Fig. 1d, which shows a differential MOKE image of a TmIG/Pt device after the application of a current pulse. The bright contrast coinciding with the Pt current line shows the region where the magnetization has switched from $-\mathbf{m}$ to $+\mathbf{m}$, demonstrating that it is possible to control the magnetization of a continuous TmIG film in a local way without altering the magnetic moments of the surroundings. Only in the presence of an out-of-plane field, or for a significant Oersted field and Joule heating produced by an intense current pulse, the switched magnetic domain may extend beyond the Pt line (see Supplementary Note 4). Such local control of the magnetization is unique to magnetic insulators due to the confinement of the current in the metal overlayer. As discussed further below, we also find that the surrounding magnetic medium influences the switching dynamics underneath the $\mathrm{Pt}$ current line. This is seen by the fact that a larger current is required for inducing down-to-up switching relative to up-todown switching when starting from a homogenously magnetized TmIG film pointing down (Fig. 1c).

Chiral DWs in TmIG revealed by scanning NV magnetometry. As SOT-induced switching is strongly dependent on the DW structure $^{12-15}$, we use scanning NV magnetometry to reveal the DW magnetization profile in both TmIG and TmIG/Pt layers. The technique is based on a single NV defect located at the apex of a diamond tip, which senses the magnetic stray field $B_{\mathrm{NV}}(X, Y)$ emanating from a magnetic surface with high spatial resolution (Fig. 2a) ${ }^{17,35,36}$. Figure $2 \mathrm{~b}$ shows $B_{\mathrm{NV}}(X, Y)$ of the TmIG film measured in a region where a DW intersects an area partially covered by Pt. From this measurement, we reconstruct the out-ofplane component of the surface magnetization $M_{Z}(X, Y) t$ (see Methods), as shown in Fig. 2c. Although the DW runs continuously across the Pt edge, the line scans of $B_{\mathrm{NV}}(X, Y)$ shown in Fig. 2d, e reveal that the DW structure changes going from TmIG/Pt to TmIG. In order to extract the magnetization profile of the DW from these measurements, we fit $B_{\mathrm{NV}}(X, Y)$ by assuming that the magnetization components in the rotated coordinate system $X Y Z$ (Fig. 2a) vary as ${ }^{17,33}$

$$
\begin{aligned}
& M_{X}(X)=M_{\mathrm{s}} \frac{\cos \psi}{\cosh \left(\frac{X}{\Delta_{\mathrm{DW}}}\right)}, \\
& M_{Y}(X)=0, \\
& M_{Z}(X)=-M_{\mathrm{s}} \tanh \left(\frac{X}{\Delta_{\mathrm{DW}}}\right),
\end{aligned}
$$

where $\psi$ defines the angle of the in-plane magnetization direction 
a

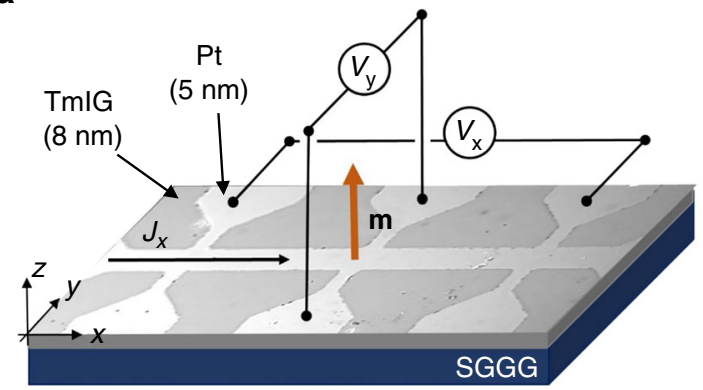

C

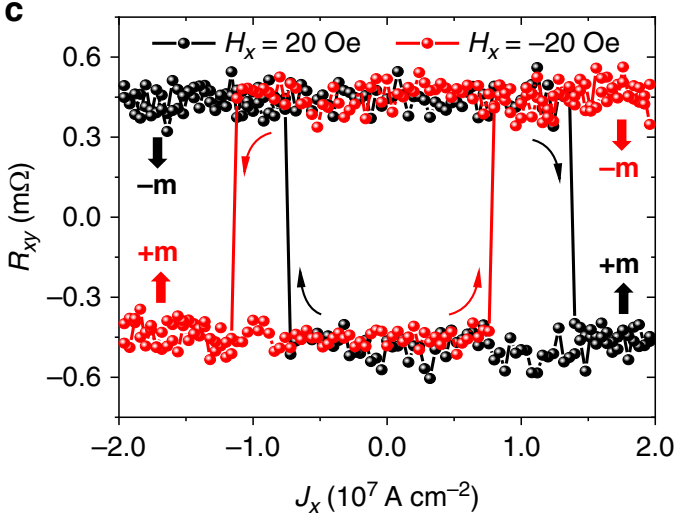

b

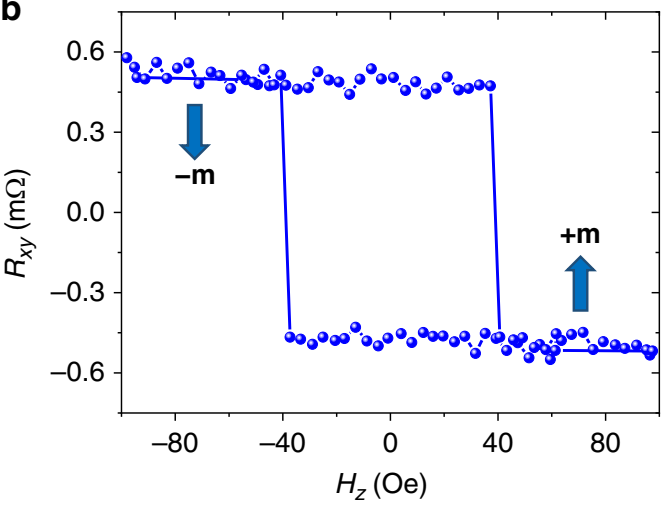

d

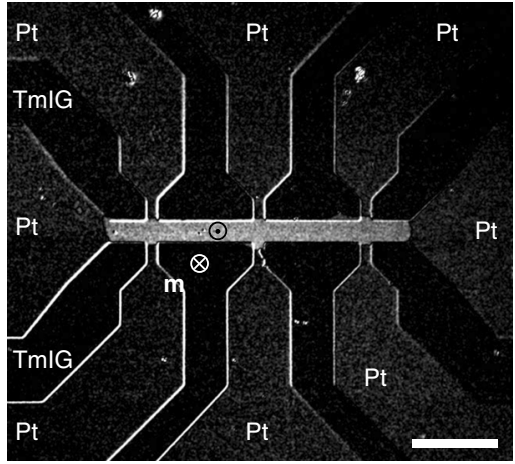

Fig. 1 Device schematics and local switching of TmIG. a Optical image of a Pt Hall bar patterned on TmIG with superposed electric wiring, coordinate system, and magnetization vector $\mathbf{m}$. $\mathbf{b}$ Hall resistance $R_{x y}$ as a function of $H_{z}$. The anomalous Hall-like signal arises from the interaction of the spin current generated in the Pt layer with the out-of-plane magnetization component $m_{z}$ of TmlG27,54, leading to a high (low) $R_{x y}$ for $-\mathbf{m}(+\mathbf{m})$. The data are shown after subtraction of a constant sample-dependent offset. c Electrical measurement of current-induced switching of TmIG $\left(t_{p}=1 \mathrm{~ms}, H_{x}=+20\right.$, black dots, and $-20 \mathrm{Oe}$, red dots). Note that, starting from a film saturated in the $\mathbf{- m}$ state, higher current densities are required to induce forward switching relative to backward switching. The same behavior is observed for $\pm H_{x}$, thus ruling out a misalignment of the sample as a possible explanation for this effect. d Wide-field differential MOKE image of a TmIG/Pt device after injection of a current pulse $\left(J_{x}=0.94 \times 10^{8} \mathrm{~A} \mathrm{~cm}-2, t_{\mathrm{p}}=150 \mathrm{~ns}, H_{x}=100 \mathrm{Oe}\right)$. The film was initially saturated in the $-\mathbf{m}$ state by applying a magnetic field $H_{z}=-100$ Oe. The bright contrast shows that only the TmIG region underneath the Pt current line has switched to $+\mathbf{m}$. Scale bar, $40 \mu \mathrm{m}$

with respect to the $X$-axis, $\Delta_{\mathrm{DW}}$ is the DW width, and $M_{\mathrm{s}}$ the saturation magnetization. Figure $2 \mathrm{~d}$, e compares representative $B_{\mathrm{NV}}(X, Y)$ line profiles for TmIG/Pt and TmIG together with the stray field profile of a pure Bloch wall $\left(\psi=90^{\circ}\right)$, a left Néel wall $\left(\psi=180^{\circ}\right)$, and a right Néel wall $\left(\psi=0^{\circ}\right)$. The best fits of the $B_{\mathrm{NV}}(X, Y)$ line profiles give $\psi=(116 \pm 33)^{\circ}$ and $\psi=(173 \pm 17)^{\circ}$, corresponding to an intermediate left-handed Néel-Bloch wall for TmIG/Pt and a left-handed Néel wall for TmIG, respectively. The $\mathrm{DW}$ widths are $\Delta_{\mathrm{DW}}=(17 \pm 17) \mathrm{nm}$ and $\Delta_{\mathrm{DW}}=(27 \pm 6) \mathrm{nm}$ for TmIG/Pt and TmIG, respectively (see Methods). Despite the large uncertainty in $\Delta_{\mathrm{DW}}$, which is due to the weak dependence of $B_{\mathrm{NV}}$ on $\Delta_{\mathrm{DW}}$, and which prevents us to determine the relative change of DW width between TmIG/Pt and TmIG, the fits show that the DWs in $8 \mathrm{~nm}$-thick TmIG are very narrow. Measurements performed in a reference unetched TmIG layer of the same thickness showed that $\psi=180^{\circ}$ and $\Delta_{\mathrm{DW}}=(20 \pm 4) \mathrm{nm}$ (see Supplementary Note 7), which confirms the left-handed Néel chirality and the narrow width of the DWs in thin TmIG films grown on SGGG.

The change of the DW chirality from left-handed Néel to an intermediate left-handed Néel-Bloch configuration in going from TmIG to TmIG/Pt is a compelling indication of the presence of negative DMI in the bare TmIG layer, most likely due to symmetry breaking at the SGGG/TmIG interface. The deposition of $\mathrm{Pt}$ reduces the DMI, which we ascribe to the presence of positive DMI at the TmIG/Pt interface, consistently with the sign of the DMI found in metallic ferromagnetic/Pt bilayers ${ }^{14,15,17}$. These findings have important consequences for the operation of DW racetracks in magnetic insulators, because the reduced $\Delta_{\mathrm{DW}}$ favors the localization of DWs, whereas the finite DMI allows for their efficient manipulation by SOTs.

Spatially resolved switching dynamics. In order to prove this last point, we investigate the switching dynamics and current-induced DW motion below the Pt line. We refer to the switching of the magnetization starting from a homogeneously magnetized TmIG layer as forward switching (domain nucleation and expansion), and to the return to a homogenous magnetic state starting from a reversed domain as backward switching (domain contraction). Figure 3a shows the relative change in the magnetization induced by a single forward switching current pulse as a function of $H_{x}$ and $J_{x}$. Within the experimental error, we find that the switching diagram is symmetric upon inversion of $H_{x}$ or $J_{x}$, indicating that the SOT efficiency is the same for up-to-down and down-to-up switching and independent on the current direction. Distinct to electrical reading $27-29$, which is only sensitive to the magnetic moments in the vicinity of the Hall cross (Fig. 1b, $c$ and Supplementary Note 5), MOKE measurements reveal that for a wide range of $H_{x}$ and $J_{x}$ only partial switching is achieved. We thus study the distribution and evolution of reversed magnetic 
a

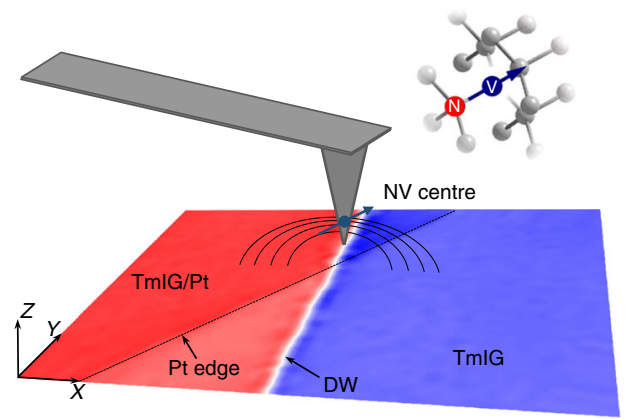

b
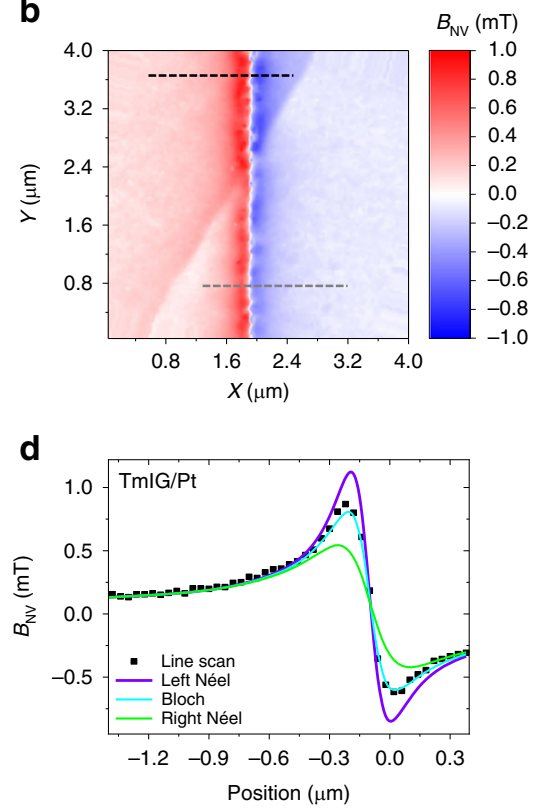

C

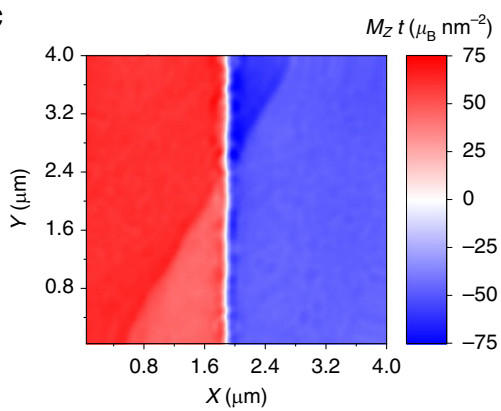

e

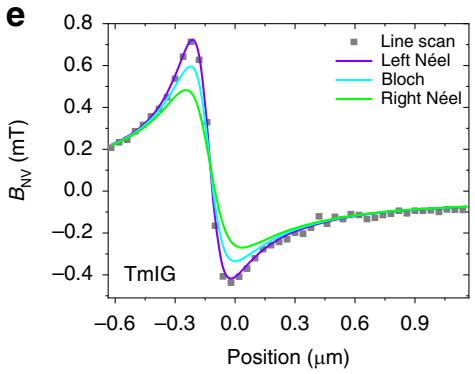

Fig. 2 Domain wall structure and chirality in TmIG and TmIG/Pt measured by scanning NV magnetometry. a Schematic of the NV probe and sample. The color code represents the out-of-plane component of the surface magnetization of the film, with red (blue) corresponding to areas of opposite magnetization. A DW crosses the TmIG/Pt and TmIG regions. The inset shows a NV center within the diamond lattice and the corresponding spin quantization axis. b Stray field $B_{\mathrm{NV}}(X, Y)$ measured by scanning the diamond tip over the sample surface shown in $\mathbf{a}$. c Reconstructed out-of-plane magnetic surface map $M_{Z}(X, Y) t$ from the data shown in $\mathbf{b}$, where $t$ is the thickness of the TmIG layer (see Methods) and $\mu_{\mathrm{B}}$ the Bohr magneton. The difference in the measured surface magnetization $M_{Z} t=(66.8 \pm 1.5) \mu_{\mathrm{B}} \mathrm{nm}^{-2}$ in TmIG/Pt and $M_{Z} t=(39.5 \pm 2.2) \mu_{\mathrm{B}} \mathrm{nm}^{-2}$ in TmlG is attributed to both the etching process and to proximity-induced polarization of the Pt layer (see Supplementary Notes 2 and 7). d, e Line scans of $B_{\mathrm{NV}}$ along the dashed lines indicated in b (square dots). The solid lines are calculated $B_{\mathrm{NV}}$ profiles using Eqs. (1-3) assuming pure Bloch ( $\psi=90^{\circ}$, cyan), left-handed Néel ( $\psi=180^{\circ}$, violet), and right-handed Néel ( $\psi=0^{\circ}$, green) DW structures for comparison with the measured stray field profiles. $M_{\mathrm{s}} t$ and $\Delta_{\mathrm{DW}}$ are set from the fits of the $B_{\mathrm{NV}}(X, Y)$ map. The fits give $\psi=(116 \pm 33)^{\circ}$ and $\Delta_{\mathrm{DW}}=(17 \pm 17) \mathrm{nm}$ for TmIG/Pt, and $\psi=(173 \pm 17)^{\circ}$ and $\Delta_{\mathrm{DW}}=(27 \pm 6) \mathrm{nm}$ for TmIG

domains induced by a sequence of current pulses. As we expect an influence of the surrounding TmIG on the magnetization dynamics underneath the Pt current line (Fig. 1c) and because the switching process is symmetric upon inverting both $\mathbf{m}$ and $\mathbf{H}^{4,8}$, we investigate the forward and backward switching processes for one fixed initial state of the film $(-\mathbf{m})$.

Figure $3 b$, c shows two representative sequences of differential MOKE images taken during forward switching and backward switching, respectively. For each case, we compare the combinations of field and current that allow for domain nucleation and expansion $\left( \pm H_{x}, \pm J_{x}\right)$ and domain contraction $\left( \pm H_{x}, \mp J_{x}\right)$. These images reveal that forward switching occurs via nucleation of a reversed domain at a defect site (as confirmed by a series of repetitions) and subsequent domain expansion along the $\mathrm{Pt}$ current line, with comparable speeds for both DWs on the leftand right-hand sides of the domain. Backward switching takes place by pushing the outer DWs towards the center of the domain. Similar dynamics-for either nucleation and expansion or contraction-is observed upon inverting $H_{x}$ and $J_{x}$. The different timescales of the switching processes (see Fig. 3b, c) indicate that domain contraction is significantly faster than domain expansion. We attribute this asymmetry to the tendency of the reversed domain to shrink in order to reduce the DW surface tension ${ }^{37,38}$. The latter is proportional to the DW length and can thus significantly offset the balance of SOT, pinning potential, and demagnetizing field. Accordingly, we find that the minimum pulse length required to induce DW motion upon contraction is much smaller than for expansion (Fig. 3d).

DW velocity and DMI. Measurements of the DW velocity $v_{\mathrm{DW}}$ are reported in Fig. 4 for an up-down DW as a function of $J_{x}$ and
$H_{x}$ during both domain expansion and domain contraction. $v_{\mathrm{DW}}$ is evaluated by considering the total DW displacement after a sequence of current pulses of length $t_{\mathrm{p}}$ as the DW moves along the Pt current line. As the same $t_{\mathrm{p}}$ does not allow for sampling a large $H_{x}, J_{x}$ parameter space, we used longer (shorter) pulses for smaller (larger) $H_{x}, J_{x}$ values. It is noteworthy that, up to $\left|J_{x}\right| \lesssim$ $1 \times 10^{8} \mathrm{~A} \mathrm{~cm}^{-2}$, the DW velocity remains almost constant when changing $t_{\mathrm{p}}$, indicating that it is not influenced by inertia, and that Joule heating plays a minor role on the DW velocity in this regime (see Methods and Supplementary Fig. 11). Our measurements reveal robust DW velocities of up to $\sim 200 \mathrm{~m} \mathrm{~s}^{-1}$ for domain expansion (Fig. 4a) and $\sim 400 \mathrm{~m} \mathrm{~s}^{-1}$ for domain contraction (Fig. 4b), which are comparable to the ones found in allmetallic structures under similar conditions $9,14,15$. Most remarkably, however, the DW mobility $\mu_{\mathrm{DW}}=\frac{v_{\mathrm{DW}}}{J_{x}}$ reaches values in excess of $3 \times 10^{-10} \mathrm{~m}^{3} \mathrm{~A}^{-1} \mathrm{~s}^{-1}$ for $J_{x}=5 \times 10^{7} \mathrm{~A} \mathrm{~cm}^{-2}$, which is comparable to that observed in compensated metallic ferrimagnets $^{11,39}$. In contrast, most metallic ferromagnets feature $\mu_{\mathrm{DW}}=0$ in this current range ${ }^{9,10,14,15}$.

The linear increase of $v_{\mathrm{DW}}$ with $J_{x}$ for expanding and contracting walls (Fig. 4a, b) further reveals a very low onset of the DW flow regime $\left(\lesssim 5 \times 10^{6} \mathrm{~A} \mathrm{~cm}^{-2}\right)$ compared with conventional ferromagnetic layers $9,10,14,15$. This behaviour is attributed to the reduced depinning field of TmIG, 1-2 Oe (see Supplementary Fig. 6), which is one to two orders of magnitude smaller than in metallic and semiconducting thin-film ferromagnets with perpendicular magnetic anisotropy ${ }^{40}$. Upon increasing the current, $v_{\mathrm{DW}}$ reaches a plateau between $\sim 0.5 \times 10^{8} \mathrm{~A} \mathrm{~cm}^{-2}$ and $\sim 0.9 \times 10^{8} \mathrm{~A} \mathrm{~cm}^{-2}$, followed by a further upturn. The plateau indicates the saturation of the DW velocity at $v_{\mathrm{DW}}^{\text {sat }} \approx \gamma \Delta_{\mathrm{DW}} \frac{\pi}{2}\left(H_{x}+H_{\mathrm{DMI}}\right)$, which occurs at a current density $J_{x}>>\frac{2 e \alpha \mu_{0} M_{\mathrm{s}} t}{\hbar \theta_{\mathrm{SH}}}\left(H_{x}+H_{\mathrm{DMI}}\right)$, where $\gamma$ is the 
a

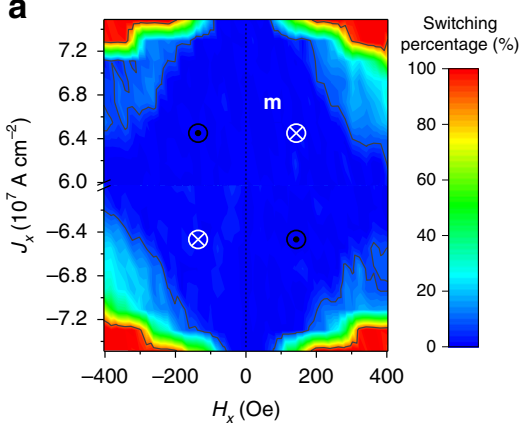

d

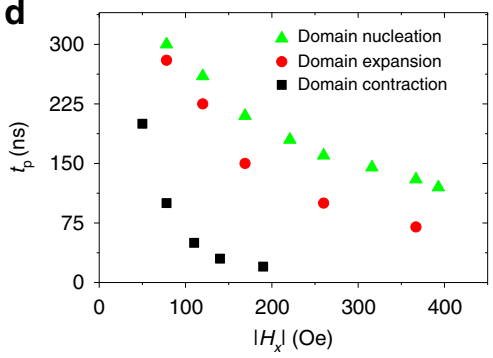

b Domain nucleation and expansion $H_{x} \rightarrow J_{x} \rightarrow$
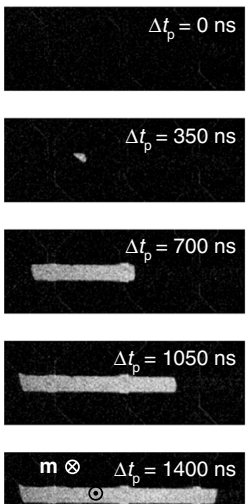
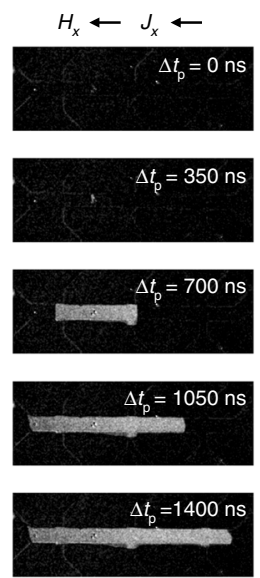

C
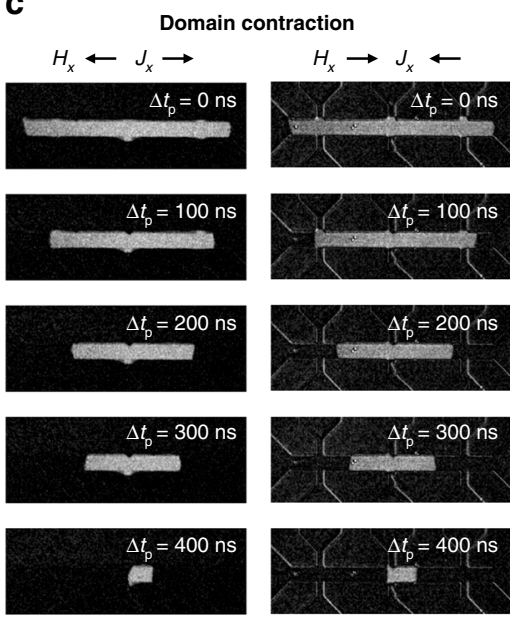

$\Delta t_{\mathrm{p}}=500 \mathrm{~ns}$

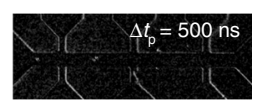

Fig. 3 Current-induced DW dynamics in embedded TmIG racetracks. a Magnetization switching diagram showing the percent change of the magnetization underneath the Pt current line as a function of $J_{x}$ and $H_{x}$. For each switching event, the initial $\mathbf{m}$ was always set fully up or down by applying an out-of-plane field $H_{z}=+100$ or -100 Oe, respectively ( $+\mathbf{m}$ for $\pm H_{x \prime} \mp J_{x}$ and $-\mathbf{m}$ for $\pm H_{z \prime} \pm J_{z}$ according to the symmetry of SOT switching). Forward switching is triggered by a single current pulse with $t_{\mathrm{p}}=500 \mathrm{~ns}$. $\mathbf{b}$ Sequence of differential MOKE images showing current-induced domain nucleation and expansion and $\mathbf{c}$ domain contraction for the different combinations of $H_{x}$ and $J_{x}$ that allow each scenario to take place. $\Delta t_{p}=N t_{p}$ is the accumulated pulse time after applying $N$ current pulses of length $t_{\mathrm{p}}$. The pulse length is $t_{\mathrm{p}}=350$ and $50 \mathrm{~ns}$, and the current density $\left|J_{x}\right|=0.65 \times 10^{8}$ and $0.75 \times 10^{8} \mathrm{~A} \mathrm{~cm}-2$ for $\mathbf{b}$ and $\mathbf{c}$, respectively. $\left|H_{x}\right|=250$ Oe in all cases. Bright and dark MOKE contrasts correspond to $+\mathbf{m}$ and $-\mathbf{m}$ states, respectively. It is noteworthy that the TmIG film not covered by Pt remains fully down magnetized. Scale bars, $20 \mu \mathrm{m}$ b, c. d Switching diagram showing the minimum pulse length $t_{\mathrm{p}}$ required to nucleate a domain starting from a fully saturated film (green triangles), to expand a domain (red circles), and to contract a domain (black squares) as a function of $\left|H_{x}\right|$. The current density of the pulses is fixed to $\left|J_{x}\right|=0.75 \times 10^{8} \mathrm{~A} \mathrm{~cm}^{-2}$. The results show that it is easier to move a DW than to nucleate a domain, and that the onset of DW motion is lower for contraction relative to expansion

gyromagnetic ratio, $\alpha$ the damping constant, $e$ the electron charge, $\hbar$ the reduced Planck constant, $\mu_{0}$ the vacuum permeability, $\theta_{\mathrm{SH}}$ the effective spin Hall angle of $\mathrm{Pt}$, and $H_{\mathrm{DMI}}$ the effective DMI field ${ }^{12,16}$. Taking $\gamma \sim 1.43 \times 10^{7} \mathrm{Oe}^{-1} \mathrm{~s}^{-1}$ (ref. ${ }^{41}$ ) and $\Delta_{\mathrm{DW}} \sim$ $20 \mathrm{~nm}$ (Fig. 2), and by approximating $H_{x}+H_{\mathrm{DMI}} \approx H_{x}=300 \mathrm{Oe}$, we find that $v_{\mathrm{DW}}^{\text {sat }} \sim 135 \mathrm{~m} \mathrm{~s}^{-1}$, which agrees well with the experimental data (Fig. 4a, b). It is noteworthy that the influence of the surrounding film on $v_{\mathrm{DW}}$ is not considered in this estimate. In fact, the DW surface tension results in a variation of the DW mobility between contracting and expanding domains by approximately a factor of 1.75 , a difference that remains constant as a function of field and current for DWs moving in the flow and saturation regimes up to $\left|J_{x}\right| \lesssim 0.9 \times 10^{8} \mathrm{~A} \mathrm{~cm}^{-2}$ (see Supplementary Fig. 12). The further increase of $v_{\mathrm{DW}}$ beyond saturation, which is typical also of metallic ferromagnets ${ }^{10,15}$, is attributed to the influence of Joule heating and Oersted field (see Supplementary Note 4 and Supplementary Figs. 11 and 12). In this high current regime $\left(\left|J_{x}\right| \lesssim 0.9 \times 10^{8} \mathrm{~A} \mathrm{~cm}^{-2}\right)$, the steeper slope of $v_{\mathrm{DW}}\left(J_{x}\right)$ for domain expansion relative to domain contraction is consistent with the longer (shorter) pulses employed for expanding (contracting) domains (see Fig. 3d), resulting in a larger (smaller) Joule heating.

In agreement with the presence of DMI inferred from the DW magnetization profile, we observe a slightly larger $v_{\mathrm{DW}}$ when the DW moves against the direction of the current (red symbols in Fig. 4). The same behaviour is also confirmed for down-up DWs (see Supplementary Fig. 13). This asymmetry, which is characteristic of chiral Néel DWs ${ }^{14,15}$, is consistent with the partially lefthanded Néel chirality derived from scanning NV magnetometry and the sign of the torques in TmIG/Pt (see Supplementary Fig. 14 for more details). Provided that the dynamics of the DWs is restricted to the flow regime, we can estimate the effective internal DMI field of the DWs by fitting $v_{\mathrm{DW}}\left(H_{x}\right)$ to a linear function and extrapolating it to $v_{\mathrm{DW}}=0$ (see ref. ${ }^{15}$ ). The fit yields $\left|H_{\mathrm{DMI}}\right|$ $\sim 12 \pm 3$ Oe (Fig. $4 \mathrm{c}, \mathrm{d}$ ), which allows us to calculate the effective DMI constant as ${ }^{9,14} \mathrm{D}=\mu_{0} H_{\mathrm{DMI}} M_{\mathrm{s}} \Delta_{\mathrm{DW}} \sim-2 \pm 2 \mu \mathrm{J} \mathrm{m}^{-2}$, where we have taken $M_{\mathrm{s}}=(6.0 \pm 1.0) \times 10^{4} \mathrm{~A} \mathrm{~m}^{-1}$ and $\Delta_{\mathrm{DW}} \sim 20 \mathrm{~nm}$. Alternatively, the DMI constant can be estimated from the DW chirality using the equation $\cos \psi=D / D_{\mathrm{c}}$, where $D_{\mathrm{c}}=$ $2 \mu_{0} M_{\mathrm{s}}^{2} t \ln 2 / \pi^{2}$ (see refs. ${ }^{42,43}$ ). This estimate gives $D \sim-2.3 \pm$ $2.6 \mu \mathrm{J} \mathrm{m}^{-2}$, in good agreement with the value obtained from the analysis of the DW velocity. From the NV measurements of the bare TmIG, we estimate that the DMI of SGGG/TmIG is $D \sim-5.3 \pm 1.8 \mu \mathrm{J} \mathrm{m}^{-2}$.

The DMI in TmIG is thus two to three orders of magnitude smaller compared with ultrathin metallic ferromagnet/Pt bilayers $^{14,15,18}$ and one to two orders of magnitude smaller than that of ferrimagnetic metal/Pt bilayers ${ }^{11}$. As the DW mobility in the flow regime is proportional to $\Delta_{\mathrm{DW}} / \alpha M_{\mathrm{s}}$, the large mobility and high DW velocities in TmIG/Pt appear as the direct consequence of the small $M_{\mathrm{s}}$ and low-damping $\alpha$ typical of garnet layers $^{31,44}$. By tuning the interfacial DMI, we anticipate that even larger $v_{\mathrm{DW}}$ may be reached at a relatively low current density.

\section{Discussion}

Our results demonstrate fast current-driven DW motion in a magnetic insulator and reveal the internal DW structure of thin garnet layers. The chiral Néel structure of the DWs in TmIG indicates that oxide interfaces support a finite DMI even in the 

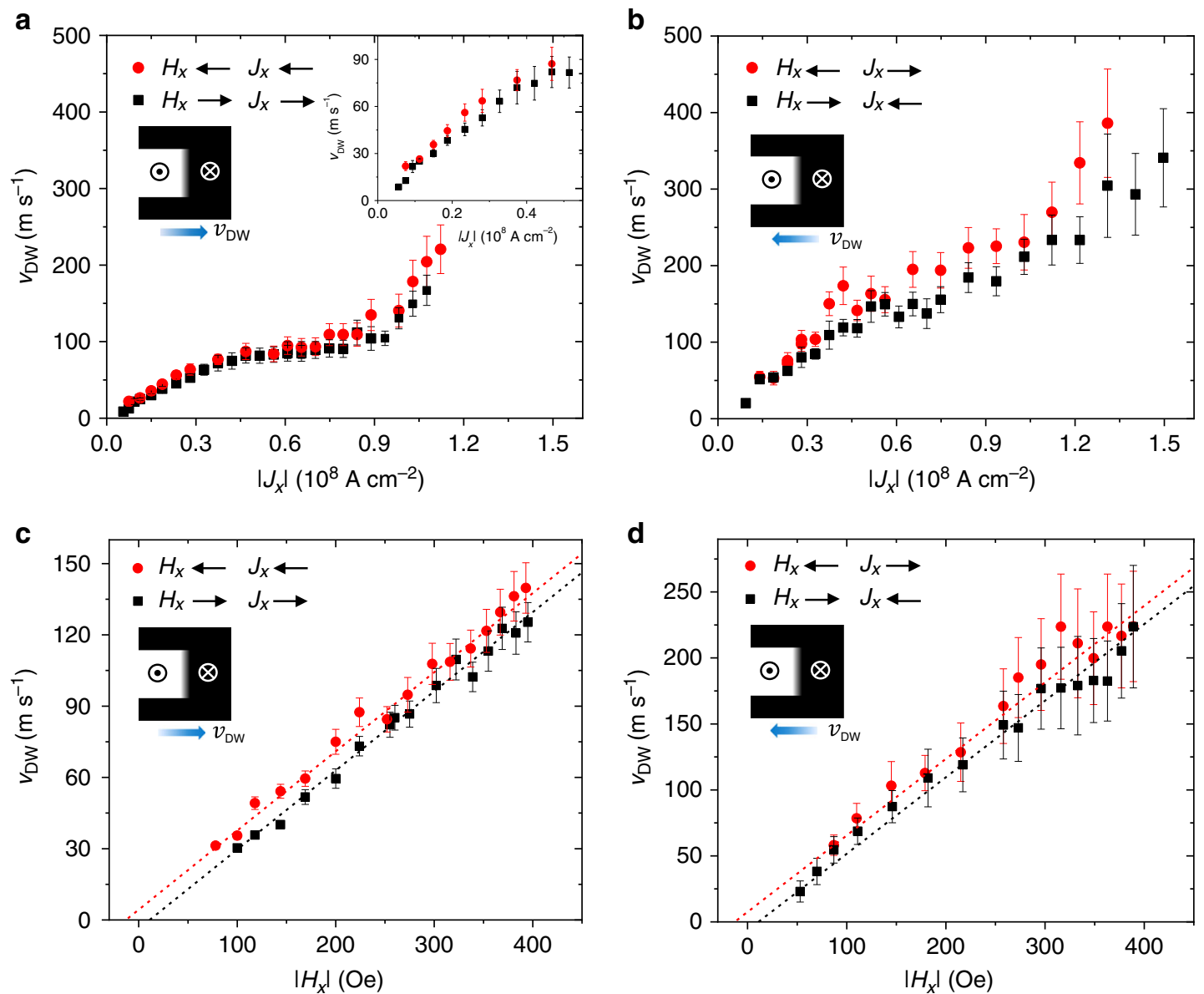

Fig. 4 DW velocity upon expansion and contraction of domains in TmIG/Pt surrounded by uniformly magnetized TmIG. a $v_{D W}$ of an up-down DW (corresponding to $\mathbf{a}+\mathbf{m}$ domain in a $-\mathbf{m}$ medium) as a function of $J_{x}$ upon expansion and $\mathbf{b}$ contraction. The magnetic field is set to $\left|H_{x}\right|=300$ Oe. The inset in a shows an enlarged view of $v_{D W}$ in the flow regime at low current density. c $v_{D W}$ of an up-down DW as a function of $H_{x}$ upon expansion and d contraction. The current density is fixed at $\left|J_{x}\right|=0.75 \times 10^{8} \mathrm{~A} \mathrm{~cm}^{-2}$ in either case. DWs move faster when $J_{x}$ is opposite to $v_{D W}$ (red symbols), indicating the presence of a weak DMI favoring left-handed Néel chiral DWs. Dashed lines in either $\mathbf{c}$ or $\mathbf{d}$ are linear fits to the experimental data assuming the same slope of $v_{\mathrm{DW}}\left(H_{x}\right)$ for both polarities of $J_{x}$ and opposite magnetic field at which $v_{\mathrm{DW}}$ crosses zero, which allows evaluating the effective DMI field $\left|H_{\mathrm{DMI}}\right| \sim 12$ \pm 3 Oe (see ref. ${ }^{15}$ ). The error bars account for the uncertainty in estimating $v_{D W}$ from each measurement

absence of heavy metal layers, which makes it possible, in principle, to stabilize nontrivial topological configurations in centrosymmetric insulating magnetic thin films, such as spin spirals and skyrmions. The low current threshold for DW flow and the large DW mobility, combined with the possibility of defining DW racetracks embedded in a continuous magnetic medium, make TmIG extremely attractive for spintronic applications. Local control of the magnetization is unique to magnetic insulators, which opens the possibility of printing arbitrary circuit paths enabling, for instance, the implementation and in-situ reconfiguration of synthetic magnetic structures with tailored magnonic bands ${ }^{21,45}$ and nanomagnonic waveguides ${ }^{46,47}$. Finally, we note that recent reports also demonstrate fast current-induced DW motion in TmIG/Pt at zero field ${ }^{48}$ as well as the emergence of a finite topological Hall effect above room temperature ${ }^{49}$. These works further prove the potential of hybrid magnetic insulator/ metal heterostructures for stabilizing and manipulating chiral magnetic textures by proximity charge currents.

\section{Methods}

Films growth and devices fabrication. The TmIG thin films were grown by pulsed laser deposition on (111)-oriented $\mathrm{Gd}_{3} \mathrm{Sc}_{2} \mathrm{Ga}_{3} \mathrm{O}_{12}$ substrates (lattice constant $a=12.56 \AA)$ to achieve high tensile strain $(\sim 2 \%)$, which promotes perpendicular magnetic anisotropy ${ }^{50}$. The substrate temperature was $650^{\circ} \mathrm{C}$, the oxygen pressure was $0.2 \mathrm{mbar}$, whereas the laser fluence and repetition rate were set to
$1.35 \mathrm{~J} \mathrm{~cm}^{-2}$ and $8 \mathrm{~Hz}$, respectively. After deposition, the samples were cooled in 200 mbar oxygen at a rate of $-10 \mathrm{~K} / \mathrm{min}$. To ensure a high quality of the TmIG/Pt interface, the TmIG films were directly transferred to the sputter chamber without breaking vacuum, where the Pt layer was deposited at room temperature for $3 \mathrm{~min}$ at a power of $10 \mathrm{~W}$ in $0.05 \mathrm{mbar}$ Ar. The thickness of the layers was calibrated by $\mathrm{X}$-ray reflectometry. For the sample presented in the main text, the thicknesses of TmIG and Pt were 8.3 and $5.0 \mathrm{~nm}$, respectively. Atomic force microscopy measurements of the surface topography showed a root-mean-square roughness of about $0.15 \mathrm{~nm}$ over a $\sim 5 \times 5 \mu \mathrm{m}^{2}$ area (see Supplementary Fig. 2). The films were magnetically characterized in a superconducting quantum interference vibration sample magnetometer system. The Pt layer was patterned into Hall bars (consisting of three Hall crosses separated by $L=50 \mu \mathrm{m}$ with a total channel length of $140 \mu \mathrm{m}$ and width $W=10 \mu \mathrm{m}$ ) by photolithography and subsequent Argon plasma etching. According to the topographic and magnetic characterization of the patterned TmIG/Pt and reference TmIG samples, we estimate that etching of Pt results also in partial etching and passivation of TmIG, leading to a reduction of the effective thickness of TmIG in the etched region by $\sim 1 \mathrm{~nm}$ (see Supplementary Notes 2 and 3 ).

Electric transport measurements. The longitudinal and transverse Hall resistances $R_{x x}=V_{x} / I_{x}$ and $R_{x y}=V_{y} / I_{x}$, respectively, were measured by applying an alternating current of amplitude $I_{x}=0.3 \mathrm{~mA}$ and frequency $f=11 \mathrm{~Hz}$, and by recording the first harmonic longitudinal $\left(V_{x}\right)$ and transverse $\left(V_{y}\right)$ voltages, as shown schematically in Fig. 1a.

MOKE measurements. We used a home-built wide-field polar MOKE microscope with Koehler illumination to measure the out-of-plane component of TmIG. As a light source, we employed a collimated light-emitting diode from Prizmatix, Ltd, model MIC-LED-455L, whose spectral emission is characterized by a maximum 
peak emission at $454 \mathrm{~nm}$, centroid at $455 \mathrm{~nm}$, and a full width at half maximum of $28 \mathrm{~nm}$. Magnetic contrast was enhanced by taking differential MOKE images, i.e., each image was subtracted by a reference image captured in a fully magnetized state. The setup was equipped with two sets of orthogonal coils for the generation of out-of-plane and in-plane magnetic fields. For the switching and DW velocity studies, current pulses were injected using an AGILENT 8114A (100V/2A) pulse generator with a $50 \Omega$ output impedance. The impedance matching with the $\mathrm{Pt}$ current line was achieved by connecting a $50 \Omega$ resistance in parallel to the current line.

The relative change in the magnetization shown in Fig. 3a was evaluated by integrating the differential MOKE signal along the Pt current line (corresponding to the bright area in Fig. 1d) after the application of a single current pulse starting from a fully magnetized state.

For the domain expansion measurements (Fig. 4a, c), an initial domain was nucleated by a single current pulse at a defect site near the center of the Hall bar. For the domain contraction experiments (Fig. 4b, d), the initial domain was generated by switching the area underneath the Pt current line with a single current pulse of $t_{\mathrm{p}}=150 \mathrm{~ns},\left|J_{x}\right|=0.94 \times 10^{8} \mathrm{~A} \mathrm{~cm}^{-2}$ and $\left|H_{x}\right|=125$ Oe, leading to a domain as the one shown in Fig. 1d.

In order to compare the DW velocities obtained for domain expansion and domain contraction, we studied the same DW moving back and forth over the same area. The case presented in Fig. 4 corresponds to an up-down DW moving between the center and the right end of the Hall bar. The DW velocity $v_{\text {Dw }}$ was evaluated by measuring the total DW displacement $\Delta x$ (as identified by direct MOKE imaging) obtained after the application of a series of $N_{\mathrm{p}}$ current pulses of width $t_{\mathrm{p}}$, yielding $v_{\mathrm{DW}}=\Delta x /\left(N_{\mathrm{p}} t_{\mathrm{p}}\right)$. The pulses were applied at a frequency of 1 $\mathrm{Hz}$, to minimize the heat load during the experiment. $v_{\mathrm{DW}}$ was found to be nearly independent of $t_{\mathrm{p}}$-only showing a slight increase of $10 \%$ or less when doubling the pulse length, which we attribute to Joule heating-indicating that the DW motion coincides with the pulse duration (see Supplementary Fig. 11 for more details).

Scanning NV magnetometry. Spatially resolved scans of the magnetic stray field produced by a DW in TmIG (see Fig. 2a) were acquired on a home-built nanoscale scanning diamond magnetometer (NSDM) microscope. Experiments were carried out in ambient environment and at zero magnetic bias field. The NSDM employed a monolithic diamond probe tip with a single NV center implanted at the apex (QZabre LLC, www.qzabre.com). The NV center spin resonance was monitored by optically detected magnetic resonance (ODMR) spectroscopy ${ }^{36,51}$ using a nearby microwave antenna $(\sim 2.9 \mathrm{GHz})$ for spin excitation and fluorescence microscopy (532 $\mathrm{nm}$ excitation, $630-800 \mathrm{~nm}$ detection) for spin state readout. The laser power employed in our measurements was $145 \mu \mathrm{W}$ and $10 \mu \mathrm{W}$ for the sample of the main text and the TmIG $(8.5 \mathrm{~nm})$ reference sample, respectively. No influence of the illumination power on the DW structure or position was noticed over time.

To convert the spin resonance frequencies to units of magnetic field, we fitted the ODMR spectrum to a double Lorentzian and extracted the frequency difference $\Delta f$ between the resonance peaks. The detected field $B_{\mathrm{NV}}$ is then given by $\left|B_{\mathrm{NV}}\right|=\frac{\pi \Delta f}{\gamma}$, where $\gamma=2 \pi \cdot 28.0 \mathrm{GHz} / \mathrm{T}$ is the electron gyromagnetic ratio. To reestablish the relative sign of $B_{\mathrm{NV}}$, we inverted $\left(B_{\mathrm{NV}} \rightarrow-B_{\mathrm{NV}}\right)$ the image on one side of the DW (Fig. 2b). It is noteworthy that scanning NV magnetometry provides a vector projection of the magnetic field,

$$
B_{\mathrm{NV}}=\mathbf{B} \cdot \mathbf{e}_{\mathrm{NV}}=\sin \theta_{\mathrm{NV}} \cos \phi_{\mathrm{NV}} B_{x}+\sin \theta_{\mathrm{NV}} \sin \phi_{\mathrm{NV}} B_{y}+\cos \theta_{\mathrm{NV}} B_{z},
$$

because the NV center is sensitive only to fields that are parallel to its symmetry axis $\mathbf{e}_{\mathrm{NV}}$. Here, $B=\left(B_{x}, B_{y}, B_{z}\right)$ is the vector field at the position of the NV center, and $\theta_{\mathrm{NV}}$ and $\phi_{\mathrm{NV}}$ are the polar and azimuth angles of $\mathbf{e}_{\mathrm{NV}}$ in the laboratory frame (see Supplementary Fig. 15). The direction $\mathbf{e}_{\mathrm{NV}}$ is determined by the crystallographic orientation of the diamond tip and the probe arrangement in the setup (see Fig. 2a). $\theta_{\mathrm{NV}}$ and $\phi_{\mathrm{NV}}$ were calibrated by a series of ODMR measurements and confirmed by line scans. For the experiments presented in Fig. $2, \theta_{\mathrm{NV}}=(55 \pm 2)^{\circ}$ and $\phi_{\mathrm{NV}}=(83 \pm 3)^{\circ}$.

We investigated the magnetization, spin structure, and width of the DW by analyzing the local field image $B_{\mathrm{NV}}(X, Y)$ shown in Fig. $2 \mathrm{~b}$. In a first step, we fitted line cuts across the TmIG to TmIG/Pt step edge to extract the NV center stand-off distance, $d=(104 \pm 5) \mathrm{nm}$ (see Supplementary Note 7$)$. To characterize the chirality and width of the DW, we took line cuts of $B_{\mathrm{NV}}$ perpendicular to the DW as shown in Fig. $2 \mathrm{~b}$ and compared them with the analytical model given through Eq. (1). The associated magnetic stray field was obtained by forward propagation of Eq. (1) in $k$-space according to ${ }^{52,53}$,

$$
\begin{aligned}
\hat{B}_{X} & =\hat{g}\left(-k \widehat{M}_{X}+i k_{X} \widehat{M}_{Z}\right) \\
\hat{B}_{Y} & =\hat{g}\left(-k \widehat{M}_{Y}+i k_{Y} \widehat{M}_{Z}\right) \\
\hat{B}_{Z} & =\hat{g}\left(i k_{X} \widehat{M}_{X}+i k_{Y} \widehat{M}_{Y}+k \widehat{M}_{Z}\right)
\end{aligned}
$$

where hat symbols indicate Fourier transforms, $k_{X}, k_{Y}$, and $k=\left(k_{X}^{2}+k_{Y}^{2}\right)^{1 / 2}$ are the in-plane $k$-space vectors, $\hat{g}=\frac{\mu_{0} t}{2}\left(\frac{1-e^{-k t}}{k t}\right) e^{-k d}$ is the Fourier transform of the Green's function ${ }^{53}$, and $t$ is the TmIG film thickness, which is taken to be 8.3 and $7.3 \mathrm{~nm}$ for the TmIG/Pt and TmIG regions, respectively (see Supplementary
Notes 2 and 3). It is worth noting that Eq. (3) shows that only changes in $M_{Z}$ lead to a stray field, which is otherwise zero for a uniformly magnetized magnetic surface. The stray field is therefore strongest near the DW and decays to zero as one moves away from the DW. When taking line cuts along $X$ across a DW extending along $Y$, the $Y$ and $\hat{M}_{Y}$ terms become zero and Eq. (3) simplifies to $\hat{B}_{X}=\hat{g}\left(-k \hat{M}_{X}+i k_{X} \hat{M}_{Z}\right), \hat{B}_{Y}=0$, and $\hat{B}_{Z}=\hat{g}\left(i k_{X} \hat{M}_{X}+k \hat{M}_{Z}\right)$.

To extract values for $M_{\mathrm{s}}, \psi$, and $\Delta_{\mathrm{DW}}$, we fitted the experimentally measured $B_{\mathrm{NV}}$ to the analytical prediction by Eqs. (1-3), with the DW position $x=x_{0}$ as an additional fit parameter. By repeating the fitting procedure for a series of line scans, we obtained distributions for all parameters together with their means and standard deviations. A detailed description of the fitting procedure and error analysis is given in the Supplementary Note 7. It is noteworthy that we can infer $\Delta_{\text {DW }}$ values that are below the NV-to-sample distance due to the large signal-tonoise ratio in our experiments and because the stray field extends far beyond the nominal DW width (see Eq. (1)).

By assuming that the magnetization is predominantly out-of-plane, we further reconstructed the surface magnetization from the magnetic field map $B_{\mathrm{NV}}(X, Y)$ by using

$$
\hat{M}_{Z}=\frac{-\hat{B}_{\mathrm{NV}} \zeta}{g\left(i k_{X} e_{X}+i k_{Y} e_{Y}-k e_{Z}\right)},
$$

where $\zeta$ is a low-pass filter (cutoff $\lambda=d$ ) that suppressed high spatial frequencies in the image ${ }^{53}$. Independently of the thickness of the film, Eq. (4) yields the surface magnetization of the film in units of magnetic moment/area, i.e., it provides the value of $M_{Z} t$. The resultant $M_{Z}(X, Y) t$ surface map is plotted in Fig. 2c. It is noteworthy that although the magnetic domains of TmIG and TmIG/Pt are well reproduced, the reconstruction slightly overestimates $M_{Z}$ near the DW due to the left Néel character of the DW.

\section{Data availability}

The data that support the findings of this study are available from the corresponding authors on reasonable request.

Received: 19 March 2019; Accepted: 23 September 2019; Published online: 18 October 2019

\section{References}

1. Brataas, A., D. Kent, A. \& Ohno, H. Current-induced torques in magnetic materials. Nat. Mater. 11, 372-381 (2012).

2. Garello, K. et al. Symmetry and magnitude of spin-orbit torques in ferromagnetic heterostructures. Nat. Nanotechnol. 8, 587-593 (2013).

3. Manchon, A. et al. Current-induced spin-orbit torques in ferromagnetic and antiferromagnetic systems. Rev. Mod. Phys. 91, 035004 (2019).

4. Miron, I. M. et al. Perpendicular switching of a single ferromagnetic layer induced by in-plane current injection. Nature 476, 189-193 (2011).

5. Liu, L. et al. Spin-torque switching with the giant spin Hall effect of tantalum. Science 336, 555-558 (2012).

6. Garello, K. et al. Ultrafast magnetization switching by spin-orbit torques. Appl Phys. Lett. 105, 212402 (2014)

7. Fukami, S., Anekawa, T., Zhang, C. \& Ohno, H. A spin-orbit torque switching scheme with collinear magnetic easy axis and current configuration. Nat. Nanotechnol. 11, 621-625 (2016).

8. Baumgartner, M. et al. Spatially and time-resolved magnetization dynamics driven by spin-orbit torques. Nat. Nanotechnol. 12, 980-986 (2017).

9. Miron, I. M. et al. Fast current-induced domain-wall motion controlled by the Rashba effect. Nat. Mater. 10, 419-423 (2011).

10. Yang, S.-H., Ryu, K.-S. \& Parkin, S. Domain-wall velocities of up to $750 \mathrm{~m} \mathrm{~s}^{-1}$ driven by exchange-coupling torque in synthetic antiferromagnets. Nat. Nanotechnol. 10, 221-226 (2015).

11. Caretta, L. et al. Fast current-driven domain walls and small skyrmions in a compensated ferrimagnet. Nat. Nanotechnol. 13, 1154-1160 (2018).

12. Thiaville, A., Rohart, S., Jué, É., Cros, V. \& Fert, A. Dynamics of Dzyaloshinskii domain walls in ultrathin magnetic films. Europhys. Lett. 100, 57002 (2012).

13. Khvalkovskiy, A. V. et al. Matching domain-wall configuration and spin-orbit torques for efficient domain-wall motion. Phys. Rev. B 87, 020402 (2013).

14. Emori, S., Bauer, U., Ahn, S.-M., Martinez, E. \& Beach, G. S. D. Currentdriven dynamics of chiral ferromagnetic domain walls. Nat. Mater. 12, 611-616 (2013).

15. Ryu, K.-S., Thomas, L., Yang, S.-H. \& Parkin, S. Chiral spin torque at magnetic domain walls. Nat. Nanotechnol. 8, 527-533 (2013)

16. Martinez, E., Emori, S., Perez, N., Torres, L. \& Beach, G. S. D. Current-driven dynamics of Dzyaloshinskii domain walls in the presence of in-plane fields: full micromagnetic and one-dimensional analysis. J. Appl. Phys. 115, 213909 (2014). 
17. Tetienne, J.-P. et al. The nature of domain walls in ultrathin ferromagnets revealed by scanning nanomagnetometry. Nat. Commun. 6, 6733 (2015).

18. Ha Pham, T. et al. Very large domain wall velocities in $\mathrm{Pt} / \mathrm{Co} / \mathrm{GdOx}$ and $\mathrm{Pt} /$ Co/Gd trilayers with Dzyaloshinskii-Moriya interaction. Europhys. Lett. 113, 67001 (2016)

19. Torrejon, J., Martinez, E. \& Hayashi, M. Tunable inertia of chiral magnetic domain walls. Nat. Commun. 7, 13533 (2016).

20. Safeer, C. K. et al. Spin-orbit torque magnetization switching controlled by geometry. Nat. Nanotechnol. 11, 143-146 (2016).

21. Chumak, A. V., Vasyuchka, V. I., Serga, A. A. \& Hillebrands, B. Magnon spintronics. Nat. Phys. 11, 453-461 (2015).

22. Kajiwara, Y. et al. Transmission of electrical signals by spin-wave interconversion in a magnetic insulator. Nature 464, 262-266 (2010).

23. Cornelissen, L. J., Liu, J., Duine, R. A., Youssef, J. Ben \& Wees, B. Jvan Longdistance transport of magnon spin information in a magnetic insulator at room temperature. Nat. Phys. 11, 1022-1026 (2015).

24. Goennenwein, S. T. B. et al. Non-local magnetoresistance in YIG/Pt nanostructures. Appl. Phys. Lett. 107, 172405 (2015).

25. Evelt, M. et al. Emission of coherent propagating magnons by insulator-based spin-orbit-torque oscillators. Phys. Rev. Appl. 10, 041002 (2018).

26. Collet, M. et al. Generation of coherent spin-wave modes in yttrium iron garnet microdiscs by spin-orbit torque. Nat. Commun. 7, 10377 (2016).

27. Avci, C. O. et al. Current-induced switching in a magnetic insulator. Nat. Mater. 16, 309-314 (2017)

28. Avci, C. O. et al. Fast switching and signature of efficient domain wall motion driven by spin-orbit torques in a perpendicular anisotropy magnetic insulator/ Pt bilayer. Appl. Phys. Lett. 111, 072406 (2017).

29. Shao, Q. et al. Role of dimensional crossover on spin-orbit torque efficiency in magnetic insulator thin films. Nat. Commun. 9, 3612 (2018).

30. Li, P. et al. Spin-orbit torque-assisted switching in magnetic insulator thin films with perpendicular magnetic anisotropy. Nat. Commun. 7, 12688 (2016).

31. Paoletti, A. Physics of Magnetic Garnets (North-Holland Company, 1978).

32. Malozemoff, A. P. \& Slonczewski, J. C. Magnetic Domain Walls in Bubble Materials (Academic Press, 1979).

33. Hubert, A. \& Schäfer, R. Magnetic Domains: The Analysis of Magnetic Microstructures (Springer, 1998).

34. Bokov, V. A., Volkov, V. V., Petrichenko, N. L. \& Maryshko, M. Relation between relaxation losses in domain-wall motion and ferromagnetic resonance in garnet films. Phys. Solid State 40, 1377-1382 (1998)

35. Degen, C. L. Scanning magnetic field microscope with a diamond single-spin sensor. Appl. Phys. Lett. 92, 243111 (2008).

36. Schirhagl, R., Chang, K., Loretz, M. \& Degen, C. L. Nitrogen-vacancy centers in diamond: nanoscale sensors for physics and biology. Annu. Rev. Phys. Chem. 65, 83-105 (2014)

37. Bates, L. F. \& Davis, P. F. Direct measurement of domain wall energy. Proc. Phys. Soc. 74, 170-176 (1959).

38. Zhang, X. et al. Direct observation of domain-wall surface tension by deflating or inflating a magnetic bubble. Phys. Rev. Appl. 9, 024032 (2018).

39. Siddiqui, S. A., Han, J., Finley, J. T., Ross, C. A. \& Liu, L. Current-induced domain wall motion in a compensated ferrimagnet. Phys. Rev. Lett. 121, 057701 (2018).

40. Jeudy, V., Díaz Pardo, R., Savero Torres, W., Bustingorry, S. \& Kolton, A. B. Pinning of domain walls in thin ferromagnetic films. Phys. Rev. B 98, 054406 (2018).

41. Randoshkin, V. V. et al. Ferromagnetic resonance in $(\mathrm{Bi}, \mathrm{Tm})_{3}(\mathrm{Fe}$, Ga) $)_{5} \mathrm{O}_{12}(210)$ epitaxial films. Tech. Phys. 52, 495-497 (2007).

42. Je, S.-G. et al. Asymmetric magnetic domain-wall motion by the Dzyaloshinskii-Moriya interaction. Phys. Rev. B 88, 214401 (2013).

43. Gross, I. et al. Direct measurement of interfacial Dzyaloshinskii-Moriya interaction in $\mathrm{X}|\mathrm{CoFeB}| \mathrm{MgO}$ heterostructures with a scanning $\mathrm{NV}$ magnetometer $(\mathrm{X}=\mathrm{Ta}, \mathrm{TaN}$, and $\mathrm{W})$. Phys. Rev. B 94, 064413 (2016).

44. Wu, C. N. et al. High-quality thulium iron garnet films with tunable perpendicular magnetic anisotropy by off-axis sputtering - correlation between magnetic properties and film strain. Sci. Rep. 8, 11087 (2018).

45. $\mathrm{Yu}, \mathrm{H}$. et al. Approaching soft X-ray wavelengths in nanomagnet-based microwave technology. Nat. Commun. 7, 11255 (2016).

46. Wagner, K. et al. Magnetic domain walls as reconfigurable spin-wave nanochannels. Nat. Nanotechnol. 11, 432-436 (2016).

47. Haldar, A., Kumar, D. \& Adeyeye, A. O. A reconfigurable waveguide for energy-efficient transmission and local manipulation of information in a nanomagnetic device. Nat. Nanotechnol. 11, 437-443 (2016).

48. Avci, C. O. et al. Interface-driven chiral magnetism and current-driven domain walls in insulating magnetic garnets. Nat. Nanotechnol. 14, 561-566 (2019).
49. Shao, Q. et al. Topological Hall effect at above room temperature in heterostructures composed of a magnetic insulator and a heavy metal. Nat. Electron. 2, 182-186 (2019).

50. Kubota, M. et al. Systematic control of stress-induced anisotropy in pseudomorphic iron garnet thin films. J. Magn. Magn. Mater. 339, 63-70 (2013).

51. Gruber, A. et al. Scanning confocal optical microscopy and magnetic resonance on single defect centers. Science 276, 2012-2014 (1997).

52. Beardsley, I. A. Reconstruction of the magnetization in a thin film by a combination of Lorentz microscopy and external field measurements. IEEE Trans. Magn. 25, 671-677 (1989).

53. Roth, B. J., Sepulveda, N. G. \& Wikswo, J. P. Using a magnetometer to image a two-dimensional current distribution. J. Appl. Phys. 65, 361-372 (1989).

54. Chen, Y.-T. et al. Theory of spin Hall magnetoresistance. Phys. Rev. B 87, 144411 (2013).

\section{Acknowledgements}

This work was supported by ETH Zurich, the Swiss Competence Centre for Materials Science and Technology (CCMX), by the Swiss National Science Foundation under Grant Number 200020-172775 and by the European Research Council through the Advanced Grant Number 694955-INSEETO. We thank Rudolf Schäfer and Eva Grimaldi for discussions, and Kevin Chang and Jan Rhensius for help in construction of the scanning NV magnetometer, as well as for providing diamond tips. M.F. thanks ETH Zurich and CEMS at RIKEN for support of his research sabbatical. C.L.D. acknowledges funding by the Swiss National Science Foundation under Grant Numbers 200020-175600 and the NCCR QSIT, and by the European Commission through Grant Number 820394 "ASTERIQS".

\section{Author contributions}

S.V., J.S., M.T. and P.G. conceived the study. J.S., E.G. and M.T. grew the TmIG and TmIG/Pt films and characterized the magnetic and structural properties. S.V. fabricated the TmIG/Pt devices and performed and analyzed the electrical measurements. S.V., J.S. and M.M. performed the MOKE measurements and S.V. and J.S. analyzed the data. M.M., C.G. and C.N. built the wide-field MOKE setup. M.S.W., P.W. and C.L.D. built the scanning NV magnetometer and performed and analyzed the scanning NV measurements. M.T., M.F., C.L.D. and P.G. supervised the work. S.V. and P.G. wrote the manuscript. All authors contributed to the scientific discussion and manuscript revision.

\section{Competing interests}

The authors declare no competing interests.

\section{Additional information}

Supplementary information is available for this paper at https://doi.org/10.1038/s41467 019-12676-7.

Correspondence and requests for materials should be addressed to S.V., C.L.D., M.T., M.F. or P.G.

Peer review information Nature Communications thanks Chunhui Du and the other, anonymous, reviewer(s) for their contribution to the peer review of this work.

Reprints and permission information is available at http://www.nature.com/reprints

Publisher's note Springer Nature remains neutral with regard to jurisdictional claims in published maps and institutional affiliations.

Open Access This article is licensed under a Creative Commons Attribution 4.0 International License, which permits use, sharing, adaptation, distribution and reproduction in any medium or format, as long as you give appropriate credit to the original author(s) and the source, provide a link to the Creative Commons license, and indicate if changes were made. The images or other third party material in this article are included in the article's Creative Commons license, unless indicated otherwise in a credit line to the material. If material is not included in the article's Creative Commons license and your intended use is not permitted by statutory regulation or exceeds the permitted use, you will need to obtain permission directly from the copyright holder. To view a copy of this license, visit http://creativecommons.org/ licenses/by/4.0/

(C) The Author(s) 2019 